\title{
A VOCAÇÃO ECONÔMICA LOCAL COMO ANTECEDENTE À ORIENTAÇÃO PARA O MERCADO: UM ESTUDO COMPARATIVO ENTRE LOCALIDADES DO LITORAL SUL DO BRASIL \\ THE LOCAL ECONOMICAL VOCATION AS A BACKGROUNG TO THE ORIENTATION TO THE MARKET: A COMPARATIVE STUDY BETWEEN LOCALITIES OF THE SOUTH COAST OF BRAZIL
}

\section{Carlos Marcelo Ardigó}

Professor Doutor da Universidade do Vale do Itajaí - Itajaí (SC), Brasil

Data de recebimento: 01-04-2014

Fabricia Durieux Zucco

Data de aceite: 02-10-2014

Professora Doutora da Univeridade do Vale do Itajaí e da Universidade Regional de Blumenau -

Blumenau/Itajaí (SC), Brasil

\section{Marco Aurélio Petrelli}

Doutorando da Universidade Federal de Santa Catarina - Florianópolis (SC), Brasil

\section{RESUMO}

A orientação para o mercado (OPM) se consolidou ao longo das duas últimas décadas como um importante tema de pesquisa na área de gestão. Este estudo teve como objetivo analisar a relação entre a OPM e a vocação econômica local. Inicialmente, a pesquisa indicou que o construto global da OPM atingiu validade interna significante. Evidências positivas, porém de intensidade fraca, indicam que um maior nível de OPM influencia positivamente nos resultados financeiros. Contudo, os resultados sugerem que a vocação turística superior, existente nas cidades componentes de uma das amostras não refletiu em maior nível de OPM.

Palavras-chave: orientação para o mercado; vocação econômica; alojamento e alimentação; turismo.

\section{ABSTRACT}

The market orientation (OM) has consolidated its position as an important research topic in the field of management over the last two decades. This study aimed to analyze the relation between the OM and local economic vocations. Initially, the research has indicated that the global construct of the OM achieved significant internal validity. Positive evidences, yet of low intensity, indicate that a high level of OM influences positively financial results. However, the results suggest that the top tourist vocation in the components cities of the samples did not result in higher levels of OM.

Keywords: market orientation; economic vocation; food and accommodation; tourism.

Endereço dos autores:

Carlos Marcelo Ardigó

marcelo.ardigo@univali.br

Marco Aurélio Petrelli

petrelli@univali.br
Fabricia Durieux Zucco

fabriciazucco@hotmail.com 


\section{INTRODUÇÃO}

As empresas orientadas para o mercado possuem em sua característica essencial a preocupação constante em buscar as melhores formas de atender às expectativas dos clientes. As ações desenvolvidas se fortalecem como instrumento facilitador de relações de trocas satisfatórias, trazendo benefícios tanto para o público-alvo quanto para as organizações.

Entendida como a cultura organizacional que estimula comportamentos necessários para a criação de valor superior para o cliente, proporcionando vantagem competitiva para a empresa (NARVER; SLATER, 1990), a orientação para o mercado representa uma gama de processos empresariais, que incluem a compreensão de mercados-alvo e a tomada de decisões estratégicas de forma interfuncional para responder mais rápida e efetivamente aos desafios e às oportunidades ambientais (SHAPIRO, 1988; DESHPANDÉ, 1999).

Essa cultura organizacional focada no mercado concentra atenção nos consumidores e nas forças que os afetam (NARVER; SLATER, 1990; KOHLI; JAWORSKI, 1993) e agrega valor ao reunir um conjunto de recursos para explorar uma oportunidade (TAJEDDINI, 2010). Os principais achados dos inúmeros estudos do fim da década de 80 e início de 1990 correlacionam o desempenho superior das organizações em diferentes aspectos da peformance empresarial, reforçando a premissa desta filosofia entre os elementos centrais da área de marketing.

Mantovani e Borges (2006) acentuam que a orientação para o mercado influencia diretamente a eficiência empresarial, enquanto Maatoofi e Tajeddini (2011) apontam a importância da correlação dessa cultura com fatores como vendas, qualidade dos serviços e criação de redes de inovação. Para Langerak (2001), os benefícios de longo prazo, advindos de uma postura de orientação, são verdadeiramente sentidos quando há forte correlação entre as percepções da empresa e de seus públicos de interesse.

São várias as escalas utilizadas para mensurar a orientação das empresas para o mercado e avaliar o nível em que ela está incorporada na cultura organizacional e nas práticas gerenciais. As mais difundidas e utilizadas são as elaboradas por Narver e Slater (1990), Kohli, Jaworski e Kumar (1993), Deshpandé, Farley e Webster (1993) e Day (1999). Ao analisar as três primeiras, Deshpandé e Farley (1998) constatam que elas se assemelham em termos de confiabilidade e de validade externa e interna.

A escala de avaliação adotada por Hooley, Saunders e Piercy (2005) se sustenta nas proposições de Narver e Slater (1990). Os itens de avaliação propostos por esses autores se aplicam às empresas associadas ao turismo, um dos mais importantes setores econômicos do mundo, que tem crescido anualmente cerca de 7\% durante os últimos cinquenta anos (SOBRAL; PECl; SOUZA, 2006) - um crescimento que requer a adoção de estratégias para adequar o receptivo às exigências dos novos e antigos visitantes.

Diante deste contexto, este estudo tem o objetivo geral de analisar a relação entre a orientação para o mercado (OPM) nas empresas sediadas em cidades com diferentes vocações econômicas. Como objetivo específico pretende-se comparar o desempenho do nível de OPM entre as cidades integrantes da amostra 1, Balneário Camboriú (BC) e Bombinhas (BB), de economia predominantemente turística, com as cidades componentes da amostra 2, Itajaí e Navegantes, estas com a economia fortemente relacionadas a outras atividades de serviço, não possuindo o turismo e suas atividades afins como fonte principal.

Este artigo está organizado de forma a apresentar uma revisão da literatura sobre o tema, a metodologia empregada, a análise dos resultados e as considerações finais. 


\section{REFERENCIAL TEÓRICO}

O referencial teórico apresentado é resultado da interlocução com diversos autores e suas formulações sobre a temática proposta, que neste estudo foi organizada a partir da leitura de múltiplas fontes. São abordados conceitos e noções referentes à orientação para o mercado, bem como escalas propostas para avaliação desse construto em cenários organizacionais.

\subsection{Orientação para o mercado}

A orientação para o mercado é definida por Deshpandé, Farley e Webster (1993) como um conjunto de crenças que coloca o cliente em primeiro lugar, sem ignorar o interesse de proprietários, gerentes e empregados, com objetivo de criar uma empresa rentável a longo prazo. Esse conceito inclui, segundo Kohli, Jaworski e Kumar (1993), uma prática de marketing de alta qualidade, entendendo-se o lucro em longo prazo como o resultado da oferta de produtos ou serviços que satisfaçam desejos e necessidades dos consumidores.

Apesar do cliente ser o elemento mais relevante no desenvolvimento de uma orientação para o mercado e demandar das empresas o desenvolvimento de estratégias focadas em seus consumidores (TAJEDDINI, 2010; MIDDLETON, 2002), Slater e Naver (1999) asseveram ser imprescindível a análise também dos concorrentes - afirmação compartilhada por Kohli, Jaworski e Kumar (1993), para quem a orientação caminha ao lado de fatores como a turbulência ambiental e a intensidade da concorrência.

Várias pesquisas buscam identificar qual a influência dessa orientação na lucratividade das empresas (NARVER; SLATER, 1990; SLATER; NARVER, 1994), no relacionamento entre fornecedores e revendedores (BAKER; SIMPSON; SIGUAW, 1999), na inovação de produtos (LUKAS; FERREL, 2000), na abordagem do mercado de exportação
(CADOGAN; DIAMANTOPOULOS; MORTANGES, 1999; CADOGAN; DIAMANTOPOULOS; SIGUAW, 2002). Outros autores, como Maatoofi e Tajeddini (2011) e Baker e Sinkula (2009), abordam o tema na perspectiva da inovação, de projetos arriscados e na busca de vantagem frente aos concorrentes.

A orientação da empresa para o mercado é frequentemente associada à inovação e à competitividade, como nos estudos de Day (1999), Deshpandé, Farley e Webster (1993), Kohli e Jaworski (1990), Narver e Slater (1990). Também são comuns as conexões com a aprendizagem e a disseminação de conhecimento (DAY, 1999; SLATER; NARVER，1995; NARVER; SLATER, 1990; KOHLI; JAWORSKI, 1990).

Nesse contexto ganham destaque os estudos iniciais de Kohli e Jaworski (1990), que consideram fundamentais as ações de geração de inteligência e marketing, sua disseminação por toda a empresa e a subsequente resposta à inteligência gerada. Narver e Slater (1990) pontuam que a orientação para o mercado se forma a partir da união de três elementos: orientação para o cliente, orientação para o concorrente e coordenação interfuncional.

Deshpandé, Farley e Webster (1993) pautam seus estudos em uma perspectiva cultural, enfatizando a percepção do cliente sobre o nível de orientação para o mercado, enquanto Deshpandé e Farley (1998a, 1998b) focam um conjunto de atividades relacionadas à constante satisfação das necessidades dos clientes.

Para Steinman, Deshpandé e Farley (2000), conhecer a percepção dos clientes sobre esse aspecto é crucial para a empresa compreender o quanto precisa ser orientada. Webb, Webster e Krepapa (2000) compartilham dessa ideia e asseveram que a análise tomada unicamente a partir da empresa propicia uma visão unilateral do construto, ignorando aspectos vitais em termos de geração de valor para os clientes. Também Krepapa et al. (2003) consideram que o foco no consumidor, apontado por eles como um dos componentes fundamentais 
da teoria da orientação, pressupõe o reconhecimento do grau de orientação pelos clientes da empresa.

No que se refere à relação entre desempenho organizacional e a orientação para o mercado, os resultados de diversos estudos indicam haver relação positiva entre a postura de orientação para o mercado e o consequente desempenho superior das organizações, a exemplo dos trabalhos de Chang e Chen (1998), Jaworski, Kohli e Kumar (1993), Pelham (1997), Perin e Sampaio (2001), Narver e Slater (1990), Slater e Narver (1994), Van Egeren e O'Connor (1998).

Ao dar ênfase à criação e à disseminação de inteligência por toda a empresa, Kohli e Jaworski (1990, 1993) entendem que a orientação para o mercado deve necessariamente comportar a análise de fatores que alteram comportamentos e tendências capazes de influenciar os desejos e necessidades dos clientes. Isso ocorre porque o conhecimento tende a aumentar a capacidade das empresas de sentir o mercado e perceber suas mudanças, possibilitando que elas se antecipem às necessidades dos consumidores e explorem novas oportunidades de negócios (HOOLEY; SAUNDERS; PIERCY, 2005; ARTHUR, 1999).

\subsection{A escala MKTOR e o modelo de análise de Hooley, Saunders e Piercy}

A escala MKTOR, desenvolvida por Narver e Slater (1990) e apontada como uma das mais importantes e significativas por Farrel e Oczkowski (1997), é também uma das mais utilizadas em trabalhos acadêmicos sobre a orientação para o mercado.

Narver e Slater (1990) propuseram o desenvolvimento de uma medida válida para verificar a contribuição da orientação para o mercado no desempenho da empresa. Para os autores, a orientação para o mercado é uma dimensão composta por três elementos comportamentais (orientação ao cliente, orientação ao concorrente e coordenação multifuncional) e por dois critérios de decisão (foco no longo prazo e objetivo no lucro). Porém, posteriormente, seus estudos apontaram a baixa intensidade de associação com o construto dos dois últimos fatores, implicando em sua eliminação no modelo final de avaliação.

Ao analisar comparativamente as escala MKTOR E MARKOR — esta elaborada por Kohli, Jaworski e Kumar (1993) —, Gauzente (1999) observa que os componentes de orientação para o mercado são aparentemente semelhantes: ambas demonstram a preocupação dos autores com a dimensão da informação (compartilhamento e coordenação interfuncional), além da visão de futuro, mas a primeira enfoca com mais ênfase a orientação ao consumidor em relação à orientação ao competidor e à coordenação interfuncional. Fazendo o mesmo confronto, Pelhan e Wilson (1996) constatam que a escala MKTOR é mais confiável que a escala MARKOR.

Segundo Ferraresi (2010), a MKTOR foi proposta originalmente utilizando uma ponderação de sete pontos para cada variável, e posteriormente se observou sua aplicação com uma escala de cinco pontos, aparentemente sem prejuízo nos resultados. O número final que mede a intensidade da orientação para o mercado é o resultado da média simples dos escores dos três componentes, ou seja, a média de uma empresa orientada para o mercado é o resultado da média aritmética apenas das pontuações da orientação ao cliente, da orientação ao concorrente e da coordenação multifuncional (NARVER; SLATER, 1990).

Com estudos ancorados principalmente nas proposições de Narver e Slater (1990), Hooley, Saunders e Piercy (2005) argumentam que o marketing como cultura organizacional (orientação para o mercado) deve ser avaliado também no contexto de outros critérios e valores das organizações, como necessidades de acionistas e funcionários e o ambiente social em que a empresa opera. 
Os autores salientam que a gestão de marketing, "por ter a responsabilidade de gerenciar a interface entre organização e mundo exterior (tanto consumidores quanto competidores), mostra-se cada vez mais importante dentro do desenvolvimento estratégico das empresas" (HOOLEY; SAUNDERS; PIERCY, 2005, p. 35). Eles julgam fundamental identificar as forças e fraquezas da empresa com o mercado, na tentativa de gerar valor superior aos clientes, considerando que "um mundo em constante movimentação demanda um sistema de inteligência refinado" (p. 42). Pautados nesses argumentos, Hooley, Saunders e Piercy (2005) propõem uma escala para avaliação do nível de orientação para o mercado com cinco componentes ou determinantes: orientação ao cliente, orientação ao concorrente, perspectivas de longo prazo, coordenação multifuncional e cultura organizacional (explicação ampliada na seção 3.2).

\section{MÉTODO}

Quanto aos procedimentos metodológicos, este estudo caracteriza-se como uma pesquisa de levantamento do tipo survey, de caráter descritivo (Churchill, 2003). Possui como participantes proprietários, gerentes ou responsáveis pelas empresas do setor de alimentação e alojamento em quatro cidades do litoral centro-norte do estado de Santa Catarina, Brasil: Balneário Camboriú, Bombinhas, Itajaí e Navegantes. As duas primeiras cidades, devido a similaridade da atividade econômica centralizada no turismo, formam a amostra 1 do estudo. As duas últimas, compõem a amostra 2, agrupadas por sua caracterísica econômica voltada a outras atividades de serviço, que não o turismo.

Esse agrupamento ocorreu a partir do cálculo do Quociente Locacional (QL) (BRITO; ALBUQUERQUE, 2002; DELGADO; GODINHO, 2005; CROCCO et al., 2006) desenvolvido segundo a comparação da proporção do número de empresas do setor nas cidades e no estado de Santa Catarina. Enquanto na amostra 1 o QL é de 1,7 pontos, na Amostra 2 esse resultado é 1,0 ponto. Isso significa que em Balneário Camboriú e Bombinhas o número de empresas do setor é 1,7 vezes superior à proporção encontrada no estado. Já nas cidades de Itajaí e Navegantes, a proporção é semelhante ao encontrado no estado.

\subsection{Amostra e coleta de dados}

Considerou-se como população desta pesquisa as empresas do segmento de hospedagem e alimentação situadas nas quatro cidades, independentemente do seu porte. Conforme dados do Instituto Brasileiro de Geografia e Estatística (IBGE, 2013a), em 2010 as cidades da amostra 1 e da amostra 2 possuíam respectivamente 1135 e 729 empresas que atendiam às características da população desejada.

A técnica de amostragem utilizada no estudo foi a não probabilística por conveniência, que, segundo Cooper e Schindler (2003), é empregada com o propósito de testar ou obter informação sobre determinado assunto, sem que seus resultados possam ser estendidos para o universo da população. Essa escolha ocorreu devido ao fato desta análise ser um recorte dos dados de campo de uma pesquisa mais ampla e multissetorial.

O levantamento das empresas do setor de alojamento e alimentação (código e denominação conforme CNAE-IBGE: H-55), formado pelos estabelecimentos hoteleiros e outros tipos de alojamento temporário e restaurantes e outros estabelecimentos de serviços de alimentação (IBGE, 2013b), resultou em uma amostra total de 213 empresas, referente a $11,4 \%$ do total identificado nas duas amostras. Destas, 114 empresas referem-se à amostra 1 e 99 à amostra 2, pouco mais de $9,5 \%$ e $13,6 \%$, respectivamente, do total presente em cada município. 
Nas empresas integrantes da amostra, as pesquisas foram aplicadas junto aos gestores, neste caso, proprietários, gerentes e/ou responsáveis pelas empresas, mesmo que sem cargos formais. A fase de aplicação do estudo ocorreu com o envolvimento de quatro pesquisadores em campo, devidamente treinados, procurando minimizar os erros e vieses decorrentes da fase de coleta. As pesquisas foram aplicadas ao longo do segundo e terceiro trimestres de 2012.

\subsection{Elaboração do instrumento de coleta de dados}

Para avaliar as questões referentes aos objetivos deste estudo, relacionado ao nível de OPM, foi utilizado um instrumento de coleta de dados para a mensuração dos seus determinantes, considerando a escala de avaliação sugerida por Hooley, Saunders e Piercy (2005), que inclui o cliente (cinco variáveis), concorrente (quatro variáveis) e coordenação multifuncional (quatro variáveis) - estas também sugeridas por Narver e Slater (1990). Compreende ainda o foco em uma perspectiva de longo prazo (três variáveis) e a cultura organizacional (quatro variáveis), esta última reforçando a OPM como um elemento central da cultura empresarial. No total, os vinte itens foram avaliados por meio de uma escala Likert de cinco pontos ( $1=$ discordo totalmente $/ 5=$ concordo totalmente), mais a possibilidade de registrar o valor zero como indicativo do desconhecimento da existência da ação na empresa da variável mencionada.

Foram inseridas no instrumento outras questões para caracterizar o perfil das empresas pesquisadas (número de colaboradores, tempo de atividade, presença de formalização e de área de marketing ou afins na estrutura organizacional). Essas informações são apresentadas de modo descritivo. No que compete ao desempenho, uma única questão de resposta subjetiva foi apresentada, perguntando ao responsável em que nível, entre muito inferiores (1) e muito superiores (5), estavam os resultados financeiros atuais de sua empresa em relação há quatro anos. Essa questão foi apresentada apenas àquelas empresas que já haviam sido criadas anteriormente ao período de quatro anos, que foi estabelecido como referência por ser considerado como o tempo mínimo para planejamentos de longo prazo, remetendo-se ao construto de perspectivas de longo prazo (NARVER; SLATER, 1990; HOOLEY; SAUNDERS; PIERCY, 2005).

Destaca-se que no presente estudo eliminou-se a etapa de pré-teste, uma vez que o instrumento foi previamente utilizado em pesquisa anterior específica para o setor de turismo (ARDIGÓ; ZUCCO; CARDOSO, 2013).

\subsection{Tratamento dos dados}

Os procedimentos estatísticos utilizados para a análise dos dados pesquisados envolveram testes de análise de confiabilidade, considerando sua consistência interna através do coeficiente alfa de Cronbach, mais recomendado em escalas multi-itens (COOPER; SCHINDLER, 2003), estatística descritiva e análise de correlação, utilizando para sua operacionalização o software estatístico Sphinx Plus versão 2.

Quanto à mensuração do grau de OPM, bem como de suas determinantes, os dados foram obtidos apurando o escore geral resultante da soma das respostas dadas às variáveis que as compõem e o construto maior de OPM. Essa abordagem, conhecida como summated scale, é utilizada em escalas Likert (MALHOTRA, 2001; COOPER; SCHINDLER, 2003). Neste caso, os resultados variam entre zero e 25 pontos na determinante cliente; zero a 20 na determinante concorrente, coordenação multifuncional e cultura; e na perspectiva de longo prazo de zero a 15. No construto maior da OPM, o resultado pode variar de zero a 100 pontos (HOOLEY; SAUNDERS; PIERCY, 2005). 


\section{ANÁLISE EDISCUSSÃO DOS RESULTADOS}

Nesta seção são descritos e discutidos os resultados deste estudo, iniciando com a caracterização da amostra. Prossegue-se apresentando os coeficientes de confiabilidade do construto OPM e de suas cinco dimensões, conforme o modelo proposto por Hooley, Saunders e Piercy (2005). Em seguida, é feita a análise comparativa entre as amostras 1 e 2 a partir das pontuações alcançadas nas determinantes de OPM, bem como dos segmentos originados a partir deles. Os dados apurados a partir da metodologia aplicada neste estudo foram estatisticamente trabalhados e foram reunidos em tabelas que mostram percentuais e coeficientes para melhor visualização, leitura e interpretação dos resultados.

\subsection{Caracterização da amostra}

Nas duas amostras, as empresas pesquisadas foram analisadas quanto ao tempo de atividade, número de colaboradores e estrutura organizacional formal. Em relação aos respondentes, considerou-se o cargo e nível de instrução destes. Os resultados dos 114 casos da amostra 1 e de 99 casos para a amostra 2 são apresentados na Tabela 1.

O tempo de atuação de uma empresa pode ser um indicativo de que suas atividades estão trazendo os resultados que permitem sua permanência no mercado. Na amostra 1, o período com maior concentração de participantes do estudo se deu entre sete e dez anos, representando pouco mais de $39 \%$ do total. Já na amostra 2, essa faixa de tempo de atividade foi apenas aquela representada

Tabela 1: Descrição das amostras analisadas.

\begin{tabular}{|c|c|c|}
\hline Itens & \multirow{2}{*}{$\begin{array}{c}\text { Amostra } 1 \\
\text { (114 respondentes) }\end{array}$} & \multirow{2}{*}{$\begin{array}{c}\text { Amostra } 2 \\
\text { (99 respondentes) }\end{array}$} \\
\hline Tempo de atividade & & \\
\hline Até 1 ano & $9,4 \%$ & $6,1 \%$ \\
\hline De 1 a 3 anos & $20,4 \%$ & $9,1 \%$ \\
\hline De 4 a 6 anos & $25,5 \%$ & $27,3 \%$ \\
\hline De 7 a 10 anos & $39,2 \%$ & $25,3 \%$ \\
\hline De 11 a 14 anos & $15,1 \%$ & $13,1 \%$ \\
\hline De 15 a 18 anos & $12,8 \%$ & $7,1 \%$ \\
\hline De 19 ou mais anos & $20,4 \%$ & $12,1 \%$ \\
\hline \multicolumn{3}{|l|}{ Número de colaboradores } \\
\hline Até 9 & $66,7 \%$ & $79,8 \%$ \\
\hline De 10 a 49 & $28,1 \%$ & $19,2 \%$ \\
\hline De 50 a 99 & $5,2 \%$ & $1 \%$ \\
\hline \multicolumn{3}{|c|}{ Presença de estrutura organizacional formal geral e de marketing } \\
\hline Sem estrutura formal & $71,9 \%$ & $88,9 \%$ \\
\hline Área de Marketing/Comercial/Vendas & $17,5 \%$ & $5,1 \%$ \\
\hline Somente outras áreas & $10,5 \%$ & $6,1 \%$ \\
\hline \multicolumn{3}{|l|}{ Cargo do respondente } \\
\hline Proprietário & $57 \%$ & $82,8 \%$ \\
\hline Gerente / Executivo /Supervisor & $29 \%$ & $12,1 \%$ \\
\hline Responsável sem cargo formal & $14 \%$ & $5,1 \%$ \\
\hline \multicolumn{3}{|l|}{ Nível de instrução do respondente } \\
\hline Fundamental incompleto & $3,5 \%$ & $5,1 \%$ \\
\hline Fundamental completo & $5,3 \%$ & $24,3 \%$ \\
\hline Médio completo & $54,4 \%$ & $60,6 \%$ \\
\hline Superior completo & $30,7 \%$ & $8,1 \%$ \\
\hline Pós-graduado em diferentes níveis & $6,1 \%$ & $2 \%$ \\
\hline
\end{tabular}

Fonte: Dados da pesquisa de campo. 
pelo segundo maior grupo de empresas (25,3\%), ultrapassado pelos $27,3 \%$ de empresas entre $4 \mathrm{e}$ 6 anos de atividade. Nas duas amostras, a maioria dos respondentes está concentrada igualmente na tipologia de microempresas (SEBRAE, 2013), ou seja, no grupo de empresas de serviços com até nove colaboradores, porém, em maior proporção na amostra 2 (79,8\%). As de pequeno porte representam $28 \%$ da amostra 1, contra pouco mais de $19 \%$ na amostra 2. As consideradas como de médio porte (de 50 a 99 colaboradores em empresas de serviço) são menos representativas, porém em proporção cinco vezes superior na amostra 1 do que na 2.

A formalização organizacional, de acordo com o perfil de porte das organizações estudadas, como é de se esperar, não está presente em 71,9\% e $88,9 \%$ respectivamente. Das que possuem áreas formais, as funções de marketing (e afins) se fazem presentes em 17,5\% na amostra 1 e apenas 5,1\% na amostra 2.

Em relação aos respondentes, na amostra 1,57\% eram proprietários, seguidos por $29 \%$ de cargos executivos formais. Na amostra 2 essa proporção foi muito superior, com aproximadamente $83 \%$ dos respondentes com o perfil de proprietário. Esse resultado implicou na redução de executivos formais $(12,1 \%)$ e de responsáveis sem cargos formais $(5,1 \%)$. Esse resultado provavelmente é influenciado pelo maior número de empresas sem estrutura formal estabelecida nas cidades da amostra 2. Entre os respondentes, o ensino médio completo é o nível de instrução predominante nas duas amostras (54,4\% - amostra 1; 60,6\% - amostra 2). A formação superior também é representativa na amostra 1 (30,7\%), porém este grupo é de apenas pouco mais de $8 \%$ na amostra 2 .

\subsection{Análise de confiabilidade da escala}

Os coeficientes de confiabilidade do construto global de OPM e de seus cinco determinantes, conforme modelo apresentado por Hooley, Saunders e Piercy (2005), baseado na escala de Narver e Slater (1990), são apresentados na Tabela 2. O processo permitiu alcançar evidências de precisão satisfatórias na análise do construto global de OPM no âmbito das duas amostras analisadas, uma vez que os coeficientes de confiabilidade interna dos dados (alfa de Cronbach) apresentaram valores dentro dos padrões internacionais de aceitação, superando os 0,78 pontos na amostra 1 e os 0,69 na amostra 2, acima dos 0,60 pontos, coeficiente mínimo mencionado por Churchill (2003).

Tabela 2: Resultado do coeficiente alfa de Cronbach.

\begin{tabular}{l|c|c}
\hline Item & Amostra 1 & Amostra 2 \\
\hline Orientação ao cliente & 0,6936 & 0,6631 \\
\hline Orientação ao concorrente & 0,7168 & 0,6400 \\
\hline $\begin{array}{l}\text { Orientação à perspectiva de } \\
\text { longo prazo }\end{array}$ & 0,6686 & 0,5003 \\
\hline $\begin{array}{l}\text { Orientação à coordenação } \\
\text { multifuncional }\end{array}$ & 0,3644 & 0,4839 \\
\hline $\begin{array}{l}\text { Orientação relacionada à cultura } \\
\text { organizacional }\end{array}$ & 0,5523 & 0,4832 \\
\hline $\begin{array}{l}\text { Coeficiente alfa de Cronbach no } \\
\text { total da escala }\end{array}$ & 0,7870 & 0,6921 \\
\hline
\end{tabular}

Fonte: dados da pesquisa

Avaliando cada um dos determinantes da OPM, a escala apresentou resultados de confiabilidade assimétricos na maioria dos casos, comparado os achados provenientes das amostras 1 e 2 . Inicialmente, os determinantes orientação para o cliente e para o concorrente ultrapassaram o coeficiente mínimo $(0,60)$ nos dois grupos de empresas analisadas. A perspectiva de longo prazo alcançou resultado semelhante, porém apenas na amostra 1. No caso da amostra 2, o alfa foi pouco superior aos 0,50 pontos. Já os determinantes coordenação multifuncional e cultura organizacional se apresentaram em condições de confiabilidade interna abaixo do coeficiente de corte nas duas amostras.

Pode-se aceitar como um nível mínimo ideal 0,60 de alfa de Cronbach, principalmente relacionado a estudos exploratórios em ciências sociais 
(HAIR et al., 2002). Já Davis (1964) avalia que, em amostras pequenas, coeficientes mínimos de 0,50 também são aceitáveis. Garver e Mentzer (1999) estabelecem suporte a esse nível de coeficiente, principalmente quando observados nos construtos extraídos (determinantes), ou seja, em partes do construto global. Porém, para Fornell e Larcker (1981), se menos de 0,50 da variância do erro de mensuração é capturada pelo constructo, a validade dos indicadores individuais, assim como a do construto principal, é questionável. Neste caso, essa situação ocorreu apenas em alguns dos indicadores individuais, não implicando nos resultados do construto geral.

É importante resgatar que o alfa de Cronbach é uma medida de confiança que varia entre zero e um ponto (LADEIRA, 2010) e, consequentemente, os resultados apurados representam que um nível de confiabilidade está presente, porém não significativo nos determinantes coordenação multifuncional nas duas amostras e na cultura organizacional na amostra 2.

Embora mostre baixa aceitação em alguns determinantes, acredita-se que sua baixa representatividade numérica deve merecer atenção. Os achados fornecem, portanto, suporte moderado de indicativos de que o instrumento de Hooley,
Saunders e Piercy (2005) possui confiabilidade para a medição de OPM no contexto do setor de alojamento e alimentação, implícito ao mercado turístico, como já observado no estudo anterior de Ardigó, Zucco e Cardoso (2013). A possibilidade de purificação da escala, estimando as questões que mais impactaram na redução da medida de avaliação, também pode ser considerada, contudo, evitando que essa purificação invalide sua análise (LEE; HOOLEY, 2005).

\subsection{O nível de OPM e a análise das correlações}

A orientação para o mercado, elemento central deste estudo está repercutida na cultura organizacional e representada por comportamentos e práticas gerenciais que estimulam a criação de valor superior para o cliente e a construção da vantagem competitiva (NARVER; SLATER, 1990). A incorporação dessa filosofia de gestão, segundo Hooley, Saunders e Piercy (2005) aumenta a capacidade de respostas das empresas em função das mudanças no mercado, possibilitando que elas se antecipem às necessidades dos consumidores e explorem novas oportunidades de negócios. Importante é que essa filosofia pode ser mensurada, e sua constatação, em termos de intensidade de sua presença,

Tabela 3: Nível de OPM e de suas determinantes.

\begin{tabular}{l|c|c|c}
\multicolumn{1}{c|}{ Determinantes } & $\begin{array}{c}\text { Medidas } \\
\text { estatísticas }\end{array}$ & $\begin{array}{c}\text { Amostra 1 } \\
\mathbf{n = 1 1 4} \text { respondentes }\end{array}$ & $\begin{array}{c}\text { Amostra 2 } \\
\mathbf{n}=\mathbf{9 9} \text { respondentes }\end{array}$ \\
\hline \multirow{2}{*}{ Orientação ao cliente } & Média & 21,29 & 22,95 \\
\hline \multirow{2}{*}{ Orientação ao concorrente } & Desvio padrão & 3,84 & 2,32 \\
\cline { 2 - 4 } & Média & 13,37 & 13,56 \\
\cline { 2 - 4 } Orientação à perspectiva de longo prazo & Desvio padrão & 3,95 & 12,88 \\
\cline { 2 - 4 } & Média & 10,52 & 2,13 \\
\hline \multirow{2}{*}{ Orientação à coordenação multifuncional } & Desvio padrão & 3,34 & 18,05 \\
\hline \multirow{2}{*}{ Orientação relacionada à cultura organizacional } & Média & 16,62 & 2,18 \\
\cline { 2 - 4 } & Desvio padrão & 3,14 & 17,86 \\
\hline \multirow{2}{*}{ Orientação para o mercado } & Média & 16,89 & 1,97 \\
\cline { 2 - 4 } & Desvio padrão & 2,80 & 84,63 \\
\cline { 2 - 4 } & Média & 11,71 & 7,37 \\
\hline
\end{tabular}

Fonte: Dados da pesquisa. 
pode ter utilidade de efetivo guia para um ajustamento gerencial.

Baseado no modelo de avaliação proposto pelos autores, para avaliação do nível de OPM em seu construto global e determinantes, na Tabela 3 apresentam-se os resultados do total das empresas, separados conforme as duas amostras, estabelecendo a média, e a medida de desvio padrão.

Analisados os resultados, seja na condição do construto global da OPM ou em seus cinco determinantes, as empresas pertencentes à amostra 1 apresentaram resultado inferior em relação ao identificado na amostra 2. Porém, avaliando o primeiro determinante (cliente) conforme faixa de valores de pontuação, em ambos os casos a média ficou estabelecida em um intervalo de valores definido como de alto nível de orientação, entre 20 e 25 pontos (HOOLEY; SAUNDERS; PIERCY, 2005). Em nível de orientação semelhante (alto nível) se encontram também os determinantes coordenação multifuncional e cultura organizacional, nas duas amostras, com medidas de média entre $16 \mathrm{e}$ 20 pontos.
Já no determinante orientação ao concorrente, os níveis de orientação entre as empresas das duas amostras resultaram em níveis apenas moderados, já que se situaram entre 12 e 16 pontos de 20 possíveis. O único determinante em que as amostras apresentaram resultados classificados em níveis de orientação distintos foi o da orientação para uma perspectiva de longo prazo. Neste, apurou-se um nível apenas moderado na amostra 1 com média de 10,52 pontos, abaixo de 12 pontos, valor de corte para posicionar-se como de alto nível. $\mathrm{Na}$ amostra 2, com média de 12,21 pontos, o alto nível foi alcançado.

Esse desempenho inferior na amostra 1, ou superior na amostra 2, foi fundamental para estabelecer a diferença na faixa de posição quanto ao nível de orientação. Na primeira amostra, a média do construto global da OPM ficou em 78,69 pontos, abaixo dos 80 pontos de corte (80-100 pontos), resultando em um nível definido apenas como moderado. A média identificada no total de empresas da amostra 2, de 84,63 pontos, supera a faixa de corte, repercutindo em um nível de orientação

Tabela 4: Matriz de correlação do construto de OPM e determinantes para as amostras 1 e 2.

\begin{tabular}{|c|c|c|c|c|c|c|}
\hline & $\begin{array}{l}\text { Orientação } \\
\text { ao cliente }\end{array}$ & $\begin{array}{l}\text { Orientação ao } \\
\text { concorrente }\end{array}$ & $\begin{array}{c}\text { Perspectiva } \\
\text { de longo } \\
\text { prazo }\end{array}$ & $\begin{array}{l}\text { Coordenação } \\
\text { multifuncional }\end{array}$ & $\begin{array}{c}\text { Cultura } \\
\text { organizacional }\end{array}$ & $\begin{array}{c}\text { Orientação } \\
\text { para o } \\
\text { mercado }\end{array}$ \\
\hline \multicolumn{7}{|l|}{ Amostra 1} \\
\hline Orientação ao cliente & 1,00 & & & & & \\
\hline Orientação ao concorrente & $+0,37^{\mathrm{CF}}$ & 1,00 & & & & \\
\hline Perspectiva de longo prazo & $+0,40^{\mathrm{CM}}$ & $+0,20^{\mathrm{CF}}$ & 1,00 & & & \\
\hline Coordenação multifuncional & $+0,59 \mathrm{~cm}$ & $+0,28^{\mathrm{CF}}$ & $0,51 \mathrm{~cm}$ & 1,00 & & \\
\hline Cultura organizacional & $+0,61^{\mathrm{CFF}}$ & $+0,12^{\mathrm{CN}}$ & $+0,36^{\mathrm{CF}}$ & $+0,71$ CFF & 1,00 & \\
\hline Orientação para o mercado & $+0,85_{\mathrm{CMF}}$ & $+0,56^{\mathrm{CM}}$ & $+0,64$ CFF & $+0,83 \mathrm{CMF}$ & $+0,76^{\mathrm{CFF}}$ & 1,00 \\
\hline \multicolumn{7}{|l|}{ Amostra 2} \\
\hline Orientação ao cliente & 1,00 & & & & & \\
\hline Orientação ao concorrente & $+0,44^{\mathrm{CM}}$ & 1,00 & & & & \\
\hline Perspectiva de longo prazo & $+0,26^{\mathrm{CF}}$ & $+0,04^{\mathrm{CN}}$ & 1,00 & & & \\
\hline Coordenação multifuncional & $+0,34^{\mathrm{CF}}$ & $+0,21^{\mathrm{CF}}$ & $+0,06^{\mathrm{CN}}$ & 1,00 & & \\
\hline Cultura organizacional & $+0,36^{\mathrm{CF}}$ & $+0,27^{\mathrm{CF}}$ & $+0,22^{C F}$ & $+0,42 \mathrm{CM}$ & 1,00 & \\
\hline Orientação para o mercado & $+0,76^{\mathrm{CFF}}$ & $+0,67^{\text {CFF }}$ & $+0,46 \mathrm{CM}$ & $+0,62$ CFF & $+0,68^{\mathrm{CFF}}$ & 1,00 \\
\hline
\end{tabular}

Fonte: dados da pesquisa.

Observação: CN: Correlação Negligenciável; CF: Correlação Fraca; cM: Correlação Moderada; CFF: Correlação Forte; CMF: Correlação Muito Forte (FRANZBLAU, 1958). 
estabelecido pelos autores como de alto nível de OPM. Porém, é importante frisar que, mesmo assim, a distância para os 100 pontos indica que meIhorias podem ser alcançadas.

O Ministério do Turismo (2010) entende que a análise de mercado deve consistir em uma visão abrangente dos movimentos que ocorrem no turismo, incluindo as regiões de oferta de um produto turístico, dos serviços que contribuem para o desenvolvimento do potencial de visitação e das localidades concorrentes. Para melhor entender o funcionamento do mercado, é preciso considerar ainda os diferentes atores com seus múltiplos papéis, as tendências de consumo e as demandas dos turistas que se pretende atrair. Essa necessidade é consistente com os fundamentos da OPM.

A partir das pontuações obtidas no construto global da OPM, bem como em seus cinco determinantes, para cada grupo amostral desenvolveu-se a análise da existência ou não de correlações entre cada um deles. Na Tabela 4, esses resultados são descritos por meio do coeficiente de correlação $(r)$ entre o construto e os determinantes analisados. Quanto mais próximo o r estiver de $+/-1$, mais forte será o grau de associação entre o que se pretende analisar. Porém, se o $r$ for igual à zero, a associação é nula (MCDANIEL; GATES, 2004).

Apurando esse resultado de forma qualitativa, Franzblau (1958) discorre sobre diferentes níveis para o $r$ na significância da correlação, o que auxilia em sua interpretação. Para resultados menores que 0,20 , a correlação é negligenciável; se o r estiver entre 0,20 e 0,40, a correlação é fraca; valores entre 0,40 e 0,60 representam correlação moderada; já entre 0,60 e 0,80 a correlação é considerada forte; e se o $r$ for maior que 0,80, a correlação é muito forte.

Por meio dos resultados descritos na matriz de correlação, denota-se a presença de uma correlação de sinal positivo entre o construto e determinantes analisados, porém em diferentes níveis de graus de associação, independentemente da amostra analisada. Os resultados indicam que o construto global OPM é positivo e significante com todas as suas determinantes, com $r$ mínimo de 0,46 (correlação moderada com perspectiva de longo prazo na amostra 2) ao máximo de 0,85 (correlação muito forte com a orientação ao cliente na amostra 1). A coordenação multifuncional $(0,83)$ também se destaca na amostra 1 pelo seu alto nível associativo, ou seja, quanto maior o nível de OPM, maior nível de correlação multifuncional existente, ou vice-versa, não sendo possível estabelecer relação de causa/efeito. A correlação também foi forte nos determinantes cultura organizacional $(0,76)$ e longo prazo $(0,64)$, na amostra 1 . Já na amostra 2, não ocorreram correlações em nível muito forte entre os determinantes e a OPM. Como nível forte, aponta-se os determinantes cliente $(0,76)$, concorrente $(0,67)$, coordenação multifuncional $(0,62)$ e cultura organizacional $(0,68)$. No entanto, quanto ao determinante perspectiva de longo prazo, a correlação foi apenas moderada com $r$ de 0,46.

Ao avaliar a correlação existente entre os próprios determinantes da OPM, os resultados indicaram que não há presença de correlação muito forte entre eles. Contudo, em nível forte de associação estão a cultura organizacional e a coordenação multifuncional $(0,71)$, e a primeira com a orientação ao cliente $(0,61)$, ambas as associações para a amostra 1. No caso da amostra 2, nenhuma associação forte foi identificada. Em nível moderado, a orientação ao cliente e ao concorrente $(0,44)$ e a cultura organizacional e a coordenação multifuncional $(0,42)$.

\subsection{Análise comparativa entre a OPM e desempenho financeiro}

A literatura pertinente à OPM, ao longo dos últimos vinte anos, apontou fortes indícios de que a presença dessa filosofia na cultura organizacional e sua relação com a estratégia têm repercutido positivamente nos resultados financeiros e de mercado 
em organizações de diferentes setores. Uma proposição dessa análise também foi realizada neste estudo, considerando os resultados comparativos da OPM entre as empresas do setor de alojamento e alimentação das duas amostras e seus resultados financeiros, subjetivamente analisados pelos responsáveis respondentes.

A análise prévia dos dados analisados, sobre as pontuações alcançadas no construto global da OPM, em seus determinantes para cada grupo amostral, bem como dos segmentos originados a partir desses, considerando os resultados financeiros subjetivamente analisados pelos respondentes, foram classificados em um primeiro grupo como superiores (segmento $A$ ), e em um segundo grupo como iguais e inferiores (segmento B). A soma do número de observações dos dois segmentos não é igual ao total da amostra, pois há casos em que as empresas não haviam sido criadas no período retroativo aos quatro anos, prazo definido neste estudo como parâmetro de longo prazo. Os resultados são apresentados na Tabela 5.

Como já indicado em análise anterior (Tabela 4), os resultados da amostra de empresas do setor de alojamento e alimentação da amostra 1 encontram-se em um nível de OPM levemente abaixo do ocorrido na amostra 2, classificando-as distintivamente conforme a apuração da medida de média como de nível moderado de OPM na primeira e de alto nível moderado na segunda amostra. Apesar dessa diferença, quando cruzados os resultados do construto global com a avaliação subjetiva do desempenho financeiro, os achados se apresentaram com características semelhantes independentemente da amostra avaliada, com níveis de OPM maiores entre as empresas com resultados financeiros superiores (segmentos A).

Analisando de forma individual, os resultados da OPM das empresas componentes do segmento A da amostra 1, com 80,69 pontos, posiciona-se

Tabela 5: Análise entre o nível de OPM e o desempenho financeiro para as amostras 1 e 2.

\begin{tabular}{|c|c|c|c|c|}
\hline \multirow{2}{*}{ Itens analisados } & \multicolumn{2}{|c|}{$\begin{array}{c}\text { Amostra } 1 \\
\mathrm{n}= \\
\mathbf{8 8} \text { observações }\end{array}$} & \multicolumn{2}{|c|}{$\begin{array}{c}\text { Amostra } 2 \\
\mathrm{n}=84 \text { observações }\end{array}$} \\
\hline & $\begin{array}{l}\text { Segmento } A^{x} \\
n=54\end{array}$ & $\begin{array}{l}\text { Segmento } B^{y} \\
n=34\end{array}$ & $\begin{array}{l}\text { Segmento } A^{x} \\
n=67\end{array}$ & $\begin{array}{l}\text { Segmento } B^{y} \\
n=17\end{array}$ \\
\hline \multicolumn{5}{|l|}{ Orientação ao cliente } \\
\hline Média & 21,98 & 20,79 & 23,43 & 21,18 \\
\hline Desvio padrão & 3,64 & 3,95 & 1,98 & 2,98 \\
\hline \multicolumn{5}{|c|}{ Orientação ao concorrente } \\
\hline Média & 14,00 & 12,62 & 13,69 & 11,82 \\
\hline Desvio padrão & 3,63 & 4,57 & 2,06 & 3,66 \\
\hline \multicolumn{5}{|c|}{ Orientação à perspectiva de longo prazo } \\
\hline Média & 11,04 & 10,65 & 12,54 & 11,53 \\
\hline Desvio padrão & 3,20 & 2,93 & 1,78 & 2,67 \\
\hline \multicolumn{5}{|c|}{ Orientação à coordenação multifuncional } \\
\hline Média & 16,83 & 16,79 & 18,58 & 15,88 \\
\hline Desvio padrão & 3,42 & 2,57 & 1,68 & 2,26 \\
\hline \multicolumn{5}{|c|}{ Orientação relacionada à cultura organizacional } \\
\hline Média & 16,83 & 17,47 & 18,13 & 16,88 \\
\hline Desvio padrão & 2,99 & 2,29 & 1,47 & 2,67 \\
\hline \multicolumn{5}{|c|}{ Orientação para o mercado } \\
\hline Média & 80,69 & 78,32 & 86,37 & 77,29 \\
\hline Desvio padrão & 12,35 & 11,38 & 5,08 & 10,26 \\
\hline
\end{tabular}

Fonte: Dados da pesquisa.

Notas: × Segmento A: resultados financeiros superiores; y Segmento B: Resultados financeiros iguais e inferiores. 
como de alto nível de orientação, enquanto no segmento $B$, com 78,32 pontos, o enquadramento é de nível moderado. Esse padrão de enquadramento também ocorreu na amostra 2, porém, neste caso, o segmento A se distancia mais fortemente do $B$.

Ao avaliar os determinantes, na amostra 1, apenas a cultura organizacional apresentou resultado superior no segmento $B$. Nos demais determinantes, o segmento $A$ foi o que atingiu as maiores médias, porém apenas levemente superiores, destacando-se a orientação ao concorrente. Apesar das diferenças, conforme modelo de análise de Hooley, Saunders e Piercy (2005), em todos os determinantes o resultado alcançado no segmento $A$ e $B$ da amostra 1 posiciona os dois grupos nos mesmos níveis de intensidade de orientação.

Já na amostra 2, todos os determinantes atingiram médias superiores no segmento $A$, comparados ao segmento $B$. Neste, os destaques foram os determinantes coordenação multifuncional e concorrente, ambos com diferenças mais acentuadas. Essa diferença mais acentuada fez com que houvesse diferenças nos níveis de intensidade de orientação entre os segmentos A e B. Isso ocorreu nos determinantes concorrente, perspectiva de longo prazo e coordenação multifuncional, sempre posicionando o segmento $A$ em um nível acima do alcançado pelo segmento $B$.
Fortalecendo essa análise, é apresentado na Tabela 6 o resultado do coeficiente de correlação entre a variável resultados financeiro e a OPM e suas determinantes para as duas amostras. Apesar dos indícios identificados anteriormente na avaliação de média, quando analisados os resultados conjuntamente, a correlação, apesar de positiva, não atingiu níveis fortes nas amostras 1 e 2 .

Analisados os resultados, conforme a Tabela 6, a associação entre desempenho financeiro, OPM e suas determinantes, foram pouco significativas na avaliação das duas amostras, porém presentes. $\mathrm{Na}$ amostra 1, apenas a orientação à perspectiva de longo prazo $(0,25)$ atingiu correlação positiva em nível fraco. Já na amostra 2, a correlação positiva em nível fraco ocorreu nos determinantes cliente e perspectivas de longo prazo. Todos os demais determinantes, assim como o construto global da OPM, apresentaram resultados de correlações negligenciáveis.

Esse resultado, por sua vez, apontou um direcionamento diferente àqueles relatados nos trabalhos de Narver e Slater (1990), Jaworski, Kohli e Kumar (1993), Slater e Narver (1994) Pelham (1997), Chang e Chen (1998), Van Egeren e O'Connor (1998) e Perin e Sampaio (2001), que de forma contrária apontaram correlação positiva entre a OPM e o desempenho financeiro. Porém, com exceção de Van Egeren e O'Connor

Tabela 6: Matriz de correlação do construto de OPM e determinantes e o resultado financeiro para as amostras 1 e 2 .

\begin{tabular}{|c|c|c|c|c|c|c|}
\hline Aspecto analisado & $\begin{array}{l}\text { Orientação } \\
\text { ao cliente }\end{array}$ & $\begin{array}{c}\text { Orientação } \\
\text { ao concorrente }\end{array}$ & $\begin{array}{c}\text { Perspectiva } \\
\text { de longo } \\
\text { prazo }\end{array}$ & $\begin{array}{l}\text { Coordenação } \\
\text { multifuncional }\end{array}$ & $\begin{array}{c}\text { Cultura } \\
\text { organizacional }\end{array}$ & $\begin{array}{c}\text { Orientação } \\
\text { para o } \\
\text { mercado }\end{array}$ \\
\hline \multicolumn{7}{|l|}{ Amostra 1} \\
\hline $\begin{array}{l}\text { Percepção do gestor sobre } \\
\text { o resultado financeiro }\end{array}$ & $+0,12^{\mathrm{CN}}$ & $+0,09^{C N}$ & $+0,25^{C F}$ & $+0,10^{\mathrm{CN}}$ & $+0,02^{C N}$ & $+0,17^{\mathrm{CN}}$ \\
\hline \multicolumn{7}{|l|}{ Amostra 2} \\
\hline $\begin{array}{l}\text { Percepção do gestor sobre } \\
\text { o resultado financeiro }\end{array}$ & $+0,20^{C F}$ & $+0,01 \mathrm{CN}$ & $+0,23 \mathrm{CF}$ & $+0,08^{\mathrm{CN}}$ & $+0,14^{\mathrm{CN}}$ & $+0,18^{\mathrm{CN}}$ \\
\hline
\end{tabular}

Fonte: dados da pesquisa.

Obs.: cNN: Correlação Nula; cN: Correlação Negligenciável; cF: Correlação Fraca; cM: Correlação Moderada;

CFF: Correlação Forte; cMF: Correlação Muito Forte (Franzblau, 1958). 
(1998), que analisaram a correlação entre a OPM e o desempenho em empresas de serviço, os demais não possuem relação com o segmento de hospedagem e alimentação estudado neste trabalho, também caracterizados como ofertantes de serviços

\section{CONSIDERAÇÕES FINAIS}

Para obter vantagem competitiva no seu mercado de atuação, as organizações têm investido no aperfeiçoamento de produtos e serviços, o que requer um nível cada vez mais elevado de profissionalização das atividades mercadológicas.

O objetivo geral da pesquisa foi analisar a relação entre a OPM em empresas sediadas em cidades com diferentes vocações econômicas. Após análise dos dados, foi possível indicar evidências de precisão satisfatórias na análise do construto global de OPM no âmbito das amostras estudadas, uma vez que os coeficientes de confiabilidade interna dos dados apresentaram valores dentro dos padrões internacionais de aceitação.

Resultado importante se refere ao nível de OPM e à análise das correlações nos casos das cidades com vocação turística. A média ficou estabelecida em um intervalo de valores definido como de nível moderado de OPM na composição da amostra 1 e de alto nível de OPM na amostra 2. Essa distinção é baseada nos valores alcançados, considerando a faixa de corte estabelecida pelo modelo de análise de Hooley, Saunders e Piercy (2005).

A pesquisa indicou que o construto global da OPM é positivo e significante com todas as suas determinantes, porém em diferentes níveis de graus de associação, independentemente da amostra analisada. Destacam-se os determinantes orientação para o cliente e a coordenação multifuncional com correlação muito forte, implicando no resultado do construto global da OPM. O primeiro, inclusive destaca-se nas duas amostras.

Ressalta-se também que a análise comparativa entre as duas amostras apresentou evidências de que a interferência externa da vocação turística não é um elemento antecedente que influenciou positivamente nos resultados da OPM das organizações pesquisadas, já que a amostra 1 encontra-se em um nível de OPM levemente abaixo do ocorrido na amostra 2.

Apesar dessa diferença, quando cruzados os resultados do construto global com a avaliação subjetiva do desempenho financeiro, os achados se apresentaram com características mais simétricas, com níveis de OPM maiores entre as empresas com resultados financeiros superiores ou muito superiores (segmento A), do que naquelas com desempenho financeiro igual ou inferior, tanto na amostra 1 quanto na 2.

A análise do coeficiente de correlação entre a variável resultados financeiro e a OPM e suas determinantes para as duas amostras denotou essa evidência positiva, porém com níveis de associação pouco significativos.

O presente estudo buscou ampliar o conhecimento sobre a OPM nas organizações. As implicações gerenciais referem-se à compreensão das ações de mercado direcionadas para o crescimento das empresas em regiões específicas. Contudo, dado a significativa importância do tema e do setor, este artigo representa parte dos primeiros esforços que visam investigar a orientação organizacional neste mercado. Em estudos futuros, a análise desse mercado em outras regiões, bem como referente a outras vocações econômicas, merecem maiores e diversificadas análises. 


\section{REFERENCIAS}

ARDIGÓ, C.M.; ZUCCO, F.D.; CARDOSO, O. R.. Estratégias de orientação para o mercado no setor turístico de Balneário Camboriú e Bombinhas (SC, Brasil). RBTur, v. 7, n.1, p. 168-189, 2013.

ARTHUR, L. Managing in organizations that learn. Management Learning, v. 30, n. 2, p. 251-253, 1999.

BAKER, T. L.; SIMPSON, P. M.; SIGUAW, J. A. The impact of suppliers' perceptions of reseller market orientation on key relationship constructs. Journal of the Academy of Marketing Science, v. 27, n. 1, p. 50-57, 1999.

BAKER, W. E.; SINKULA, J. M. The complementary effects of market orientation and entrepreneurial orientation on profitability in small businesses. Journal of Small Business Management, v. 47, n 4, p. 443-464, 2009.

BRITTO, J.; ALBUQUERQUE, E. M.. Clusters industriais na economia brasileira: uma análise exploratória a partir de dados da RAIS. Estudos Econômicos, v. 32, n. 1, p. 71-102, jan./mar. 2002.

CADOGAN, J. W.; DIAMANTOPOULOS, A.; SIGUAW, J. A.. Export Market-Oriented Activities: Their Antecedents and Performance Consequences. Journal of International Business Studies, v. 33, n. 3, p. 615-626, 3rd Qtr., 2002.

CHANG, T. Z.; CHEN, S. J.. Market orientation, service quality and business profitability: a conceptual model and empirical evidence. The Journal of Services Marketing, v. 12, n. 4, p. 246261, 1998

CHURCHILL, G. A. Investigación de mercados. $4^{\text {a }}$. ed. México: Thomson, 2003.
COOPER, D. R.; SCHINDLER, P. S. Métodos de pesquisa em administração. 7. ed. Porto Alegre: Bookman, 2003.

CROCCO, M. A. et al.. Metodologia de identificação de aglomerações produtivas locais. Nova economia, v. 16, n. 2: p. 211-241, maio/ago. 2006.

DAVIS, F. B. Educational measurements and their interpretation. Belmont, California: Wadsworth Publishing Co, 1964.

DAY, G.S. The market driven organization: understanding, attracting, and keeping valuable customers. New York: The Free Press, 1999.

DELGADO, A. P.; GODINHO, I. M.. Medidas de localização das actividades e de especialização regional. In: Costa, J. (Coord.). Compêndio de Economia Regional. 2 ed., Coimbra: p. 713-732, 2005.

DESHPANDÉ, R.; FARLEY, J. U.; WEBSTER, F.. Corporate culture, customer orientation, and innovativeness in Japanese firms: a quadrat analysis. Journal of Marketing, v. 57, n. 1, p. 23-27, 1993.

DESHPANDÉ, R.; FARLEY, J. U. Measuring market orientation: generalization and synthesis. Journal of Market Focused Management, v. 2, n. 3, p. 213-232, $1998 a$

The market orientation construct: correlations, culture, and comprehensiveness. Journal of Market Focused Management, v. 2, n. 3, p. 237-239, 1998b.

DESHPANDÉ, R. Developing a market orientation. Thousands Oaks: Sage Publications Inc., 1999.

FARRELL, M. A.; OCZKOWSKI, E. A. An analysis of the MKTOR and MARKOR measures of market orientation: 


\section{REFERÉNCIAS}

an Australian perspective. Marketing Bulletin, v. 8, p. 30-40, 1997.

FERRARESI, A. A. Gestão do conhecimento, orientação para o mercado, inovatividade e resultados organizacionais: um estudo em empresas instaladas no Brasil. 2010. Tese (Doutorado em Administração) - Universidade de São Paulo, São Paulo. 2010.

FORNELL, C.; LARCKER, D. F. Evaluating structural equation models with unobservable variables and measurement error. Journal of Marketing Research, v. 18, n. 1, p. 39-50, 1981.

FRANZBLAU, A. A primer of statistics for nonstatisticians. New York: Harcourt, Brace \& World, 1958.

GARVER, M. S.; MENTZER, J. T. Logistics research methods: employing structural equation modeling to test for construct validity. Journal of Business Logistics, v. 20, n. 1, p. 33-57, 1999.

GAUZENTE, C. Comparing market orientation scales: a content analysis. Marketing Bulletin, v. 10, n. 4, p. 76-82, 1999.

HAIR Jr, J.F. et al. Multivariate Data Analysis, 5 ed. Upper Saddle River, NJ: Prentice Hall, 1998.HOOLEY, G. J.; SAUNDERS, J. A.; PIERCY, N. F. Estratégia de marketing e posicionamento competitivo. 3. ed. São Paulo: Pearson Prentice Hall, 2005.

IBGE. INSTITUTO BRASILEIRO DE GEOGRAFIA E ESTATÍSTICA. Banco SIDRA. Disponível em <www. sidra.ibge.gov.br/>. Acesso em: $10 \mathrm{de} \mathrm{fev.} \mathrm{2013a.}$

IBGE. INSTITUTO BRASILEIRO DE GEOGRAFIA E ESTATÍSTICA. Classificação Nacional de Atividades Econômicas - Fiscal. Rio de Janeiro: IBGE, 2013b.
JAWORSKI, B. J.; KOHLI, A. K. Market orientation: antecedents and consequences. Journal of Marketing, v. 57, p. 53-71, 1993.

KOHLI, A. K.; JAWORSKI, B. J. Market orientation: the construct, research propositions, and managerial implications. Journal of Marketing, v. 54, n. 2, p. 1-18, 1990.

KOHLI, A. K.; JAWORSKI, B. J.; KUMAR, A. MARKOR: a measure of market orientation. Journal of Marketing Research, v. 30, n. 4, p. 467-477, 1993.

KREPAPA, A. et al. Mind the gap: An analysis of service provider versus

customer perceptions of market orientation and the impact on satisfaction. European Journal of Marketing, v. 37, n. 1/2, p. 197-218, 2003.

LADEIRA, W. J. Três décadas do modelo de Churchill: utilização da análise fatorial e do alfa de Cronbach na validação de instrumentos de coleta de dados no marketing. PMKT Ciência, v. 5, p. 40-48, 2010.

LANGERAK, F.. The relationship between customer and supplier perceptions of the manufacturer's market orientation and its business performance. International Journal of Market Research, v. 43, n. 1, p. 43-62, 2001.

LEE, N.; HOOLEY, G.. The evolution of "classical mythology" within marketing measure development. European Journal of Marketing, v. 39, n. 3/4, p. 365385, 2005.

LUKAS, B.; FERREL O. C. The effect of market orientation on product innovation. Journal of the Academy of Marketing Science, v. 28, n. 2, p. 239-247, 2000.

MAATOOFI, A. R.; TAJEDDINI, K.. Effect of market orientation and entreprenurial orientation on innovation. 


\section{REFERÉNCIAS}

Journal of Management Research, v. 11, n. 1, p. 20-30, 2011.

MALHOTRA, N. K. Pesquisa de marketing: uma orientação aplicada. 3. ed. Porto Alegre: Bookman, 2001.

MANTOVANI, D.; BORGES, C. M. Comportamento empreendedor e práticas de orientação para o mercado no contexto das pequenas e médias empresas. In: ENCONTRO NACIONAL DA ASSOCIAÇÃO NACIONAL DOS PROGRAMAS DE PÓS-GRADUAÇÃO EM ADMINISTRAÇÃO (Enanpad), 30, 2006. Salvador. Anais ... Salvador: Anpad, 2006. (CD-ROM).

MCDANIEL, C. D.; GATES, R. Pesquisa de marketing. São Paulo: Pioneira Thomson Learning, 2004.

MIDDLETON, V. T. Marketing de turismo: teoria e prática. Rio de Janeiro: Campus, 2002.

MINISTÉRIO DO TURISMO. Segmentação do turismo e o mercado. Brasília: Ministério do Turismo. 2010.

NARVER, J. C.; SLATER, S. F. The effect of a market orientation on business profitability. Journal of Marketing, v. 54, n. 4, p. 20-35, 1990.

PELHAM, A. M.; WILSON, D. T. A longitudinal study of the impact of market structure, firm structure, strategy, and market orientation culture on dimensions of small-firm performance. Journal of the Academy of Marketing Science, v. 24, n. 1, p. 27-43, 1996.

PELHAM, A. M. Mediating influences on the relationship between market orientation and profitability in small industrial firms. Journal of
Marketing Theory and Pratice, v. 5, n. 3, p. 55-76, 1997.

PERIN, M. G.; SAMPAIO, C. H. A relação entre as dimensões de orientação para mercado e a performance. In: ENCONTRO NACIONAL DOS PROGRAMAS DE PÓSGRADUAÇÃO EM ADMINISTRAÇÃO (Enanpad), 25, 2001. Campinas. Anais... Campinas: Anpad. 2001. (CD-ROM).

SEBRAE. Critérios de classificação de empresas: EI - ME - EPP. Disponível em: <http://www.sebrae-sc.com.br/ leis/default.asp?vcdtexto=4154>. Acesso em: $23 \mathrm{de} \mathrm{fev.}$ 2013.

SHAPIRO, B. P. What the hell is 'market oriented'? Harvard Business Review, v. 66, n. 6, p. 119-125, 1988.

SLATER, S. F.; NARVER, J. C.. Market orientation, customer value, and superior performance. Business Horizons, v. 37, p. 22-28, 1994.

_ Market orientation and the learning organization. Journal of Marketing, v. 59, n. 3, p. 6375, 1995.

Market oriented is more than being customerled. Strategic Management Journal, v. 20, p. 11651168, 1999.

SOBRAL, F.; PECI, A.; SOUZA, G. C. Uma análise shift-share da dinâmica do setor de turismo no Brasil: recomendações para as políticas públicas. In: ENCONTRO NACIONAL DA ASSOCIAÇÃO NACIONAL DOS PROGRAMAS DE PÓS-GRADUAÇÃO EM ADMINISTRAÇÃO (Enanpad), 30, 2006. Salvador. Anais ... Salvador: Anpad. 2006. CD-ROM.

STEINMAN, C.; DESHPANDÉ, R.; FARLEY, J. U. Beyond market orientation: when customer and suppliers 


\section{REFERÉNCIAS}

disagree. Journal of the Academy of Marketing

Science, v. 28, n. 1, p. 109-119, 2000.

TAJEDDINI, K. Effect of customer orientation and entrepreneurial orientation on innovativeness: evidence from the hotel industry in Switzerland. Tourism Management, v. 31, n. 2, p. 221-231, 2010.
VAN EGEREN, M.; O'CONNOR, S. Drivers of market orientation and performance in service firms. The Journal of Services Marketing, v. 12, n. 1, p. 39-58, 1998.

WEBB, D.; WEBSTER, C.; KREPAPA, A. An exploration of the meaning and outcomes of a costumer-defined market orientation. Journal of Business Research, v. 48, n. 2, p. 101-112, 2000. 